\title{
ICG/L-Arginine encapsulated PLGA nanoparticles-thermosensitive hydrogel hybrid delivery system for cascade cancer photodynamic-NO therapy with promoted collagen depletion in tumor tissue
}

Zhiting Sun ${ }^{1}$, Xiaoxiao Wang ${ }^{1}$, Jing Liu ${ }^{1}$, Zhihong Wang ${ }^{1}$, Weiwei Wang ${ }^{1}$, Deling Kong*1, 2, Xigang Leng*1

${ }^{1}$ Tianjin Key Laboratory of Biomaterial Research, Institute of Biomedical Engineering, Chinese Academy of Medical Sciences and Peking Union Medical College, Tianjin 300192, China

${ }^{2}$ Key Laboratory of Bioactive Materials of Ministry of Education, State Key Laboratory of Medicinal Chemical Biology, College of Life Science, Nankai University, Tianjin 300071, China 

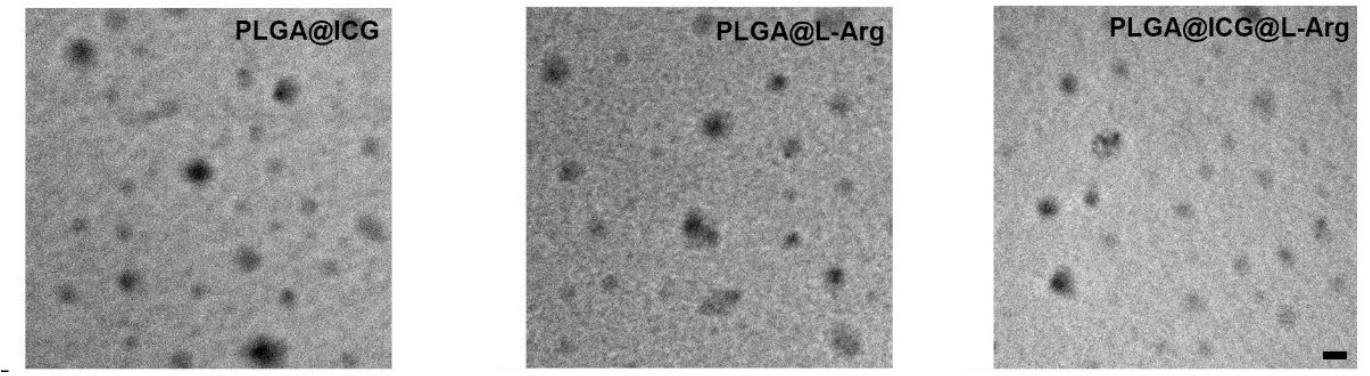

Figure S1. Characterization of PLGA@ICG@L-Arg nanoparticles. TEM images of PLGA@ICG NPs, PLGA@L-Arg NPs and PLGA@ICG@L-Arg NPs; scale bar, 200 nm.

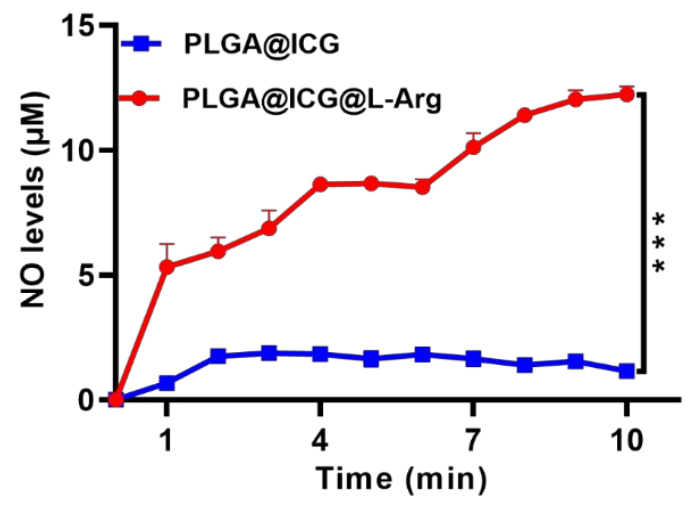

Figure S2. The release of NO in PLGA@ICG and PLGA@ICG@L-Arg group in vitro. 

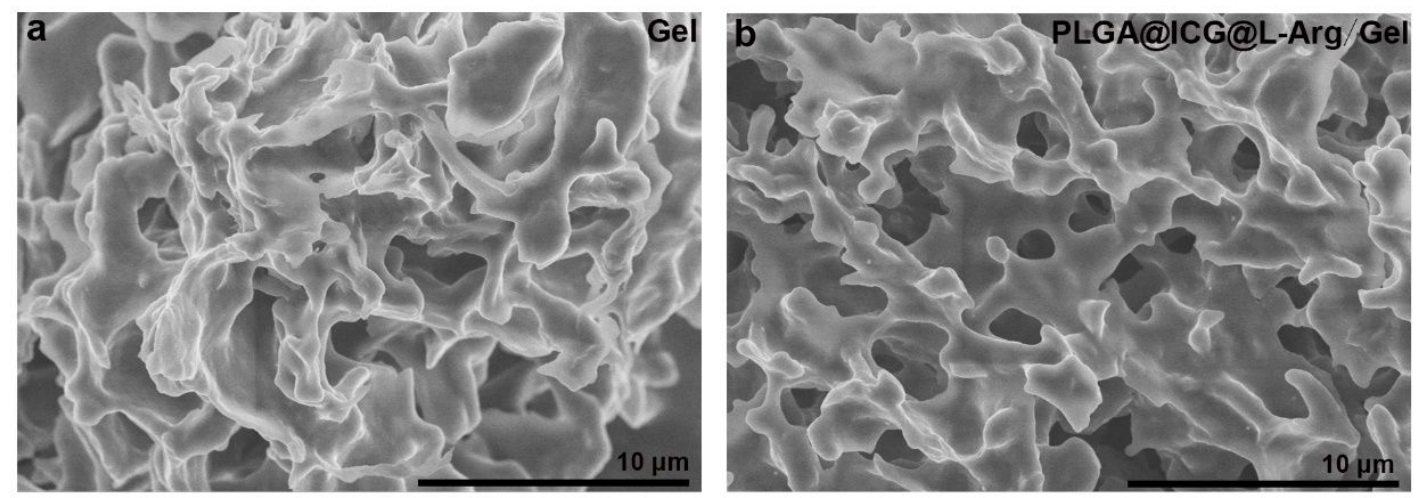

Figure S3. SEM images of hydrogel. (a) bare PCL-PEG-PCL hydrogel; (b) PLGA@ICG@L-Arg/Gel; scale bar, $10 \mu \mathrm{m}$.

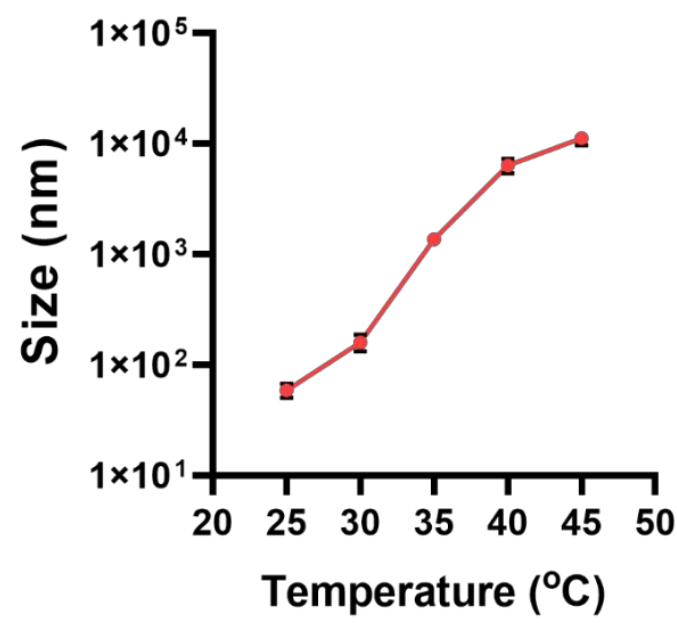

Figure S4. The size change of PLGA@ICG@L-Arg/Gel aqueous solutions (1.0wt\%) as a function of temperature. 
PLGA
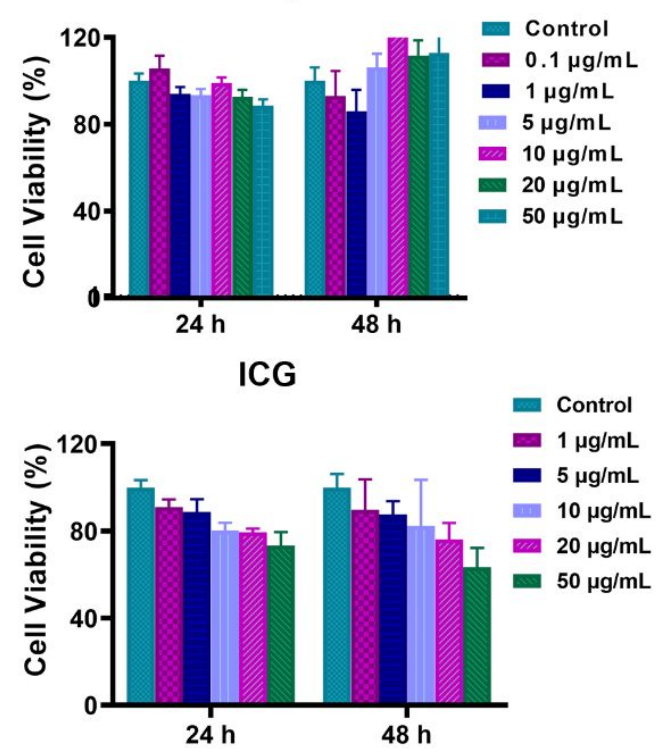

L-Arginine
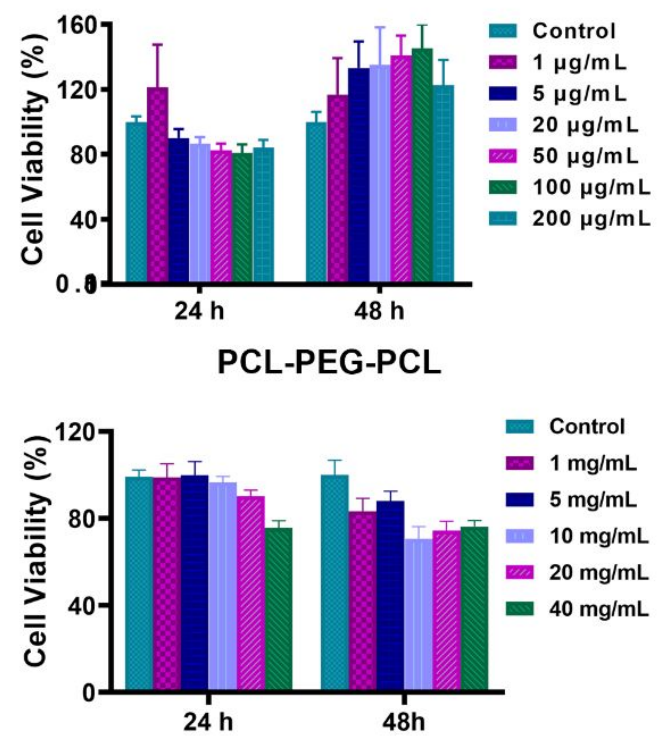

Figure S5. In vitro cell viability of 4T1 cells after treated with ICG, L-Arg, PLGA, and PCL-PEG-PCL via CCK- 8 assay.

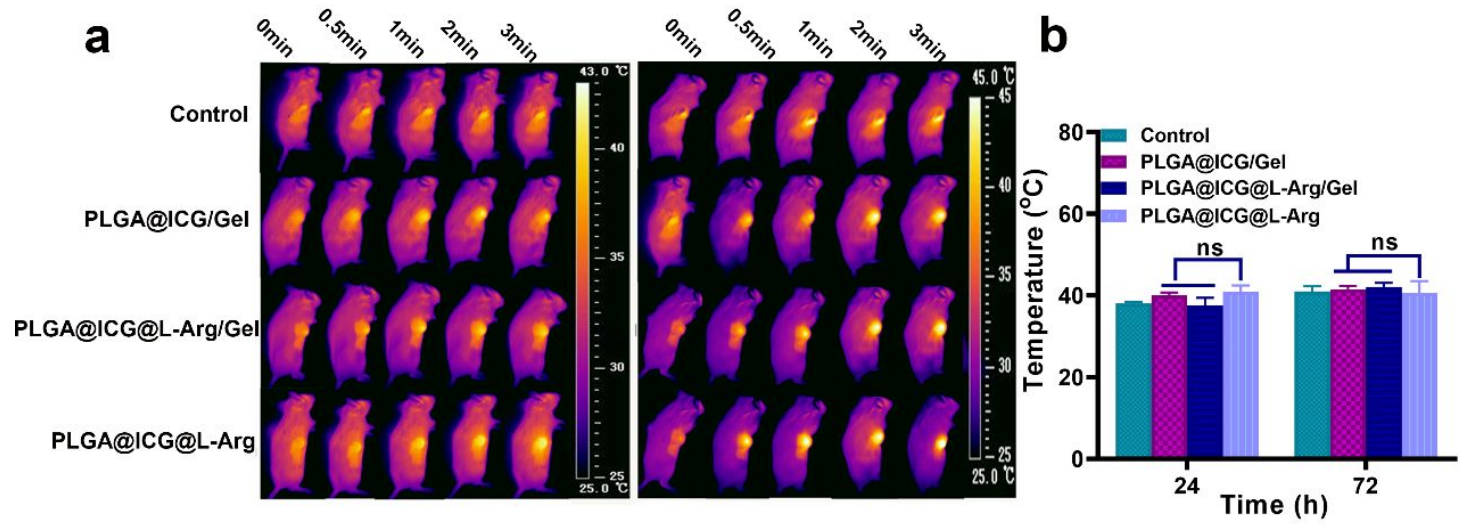

Figure S6. (a) Thermographs of tumor site in different groups under NIR irradiation at

$808 \mathrm{~nm}$ captured at preset points. (b) Quantitative statistic of temperature. 


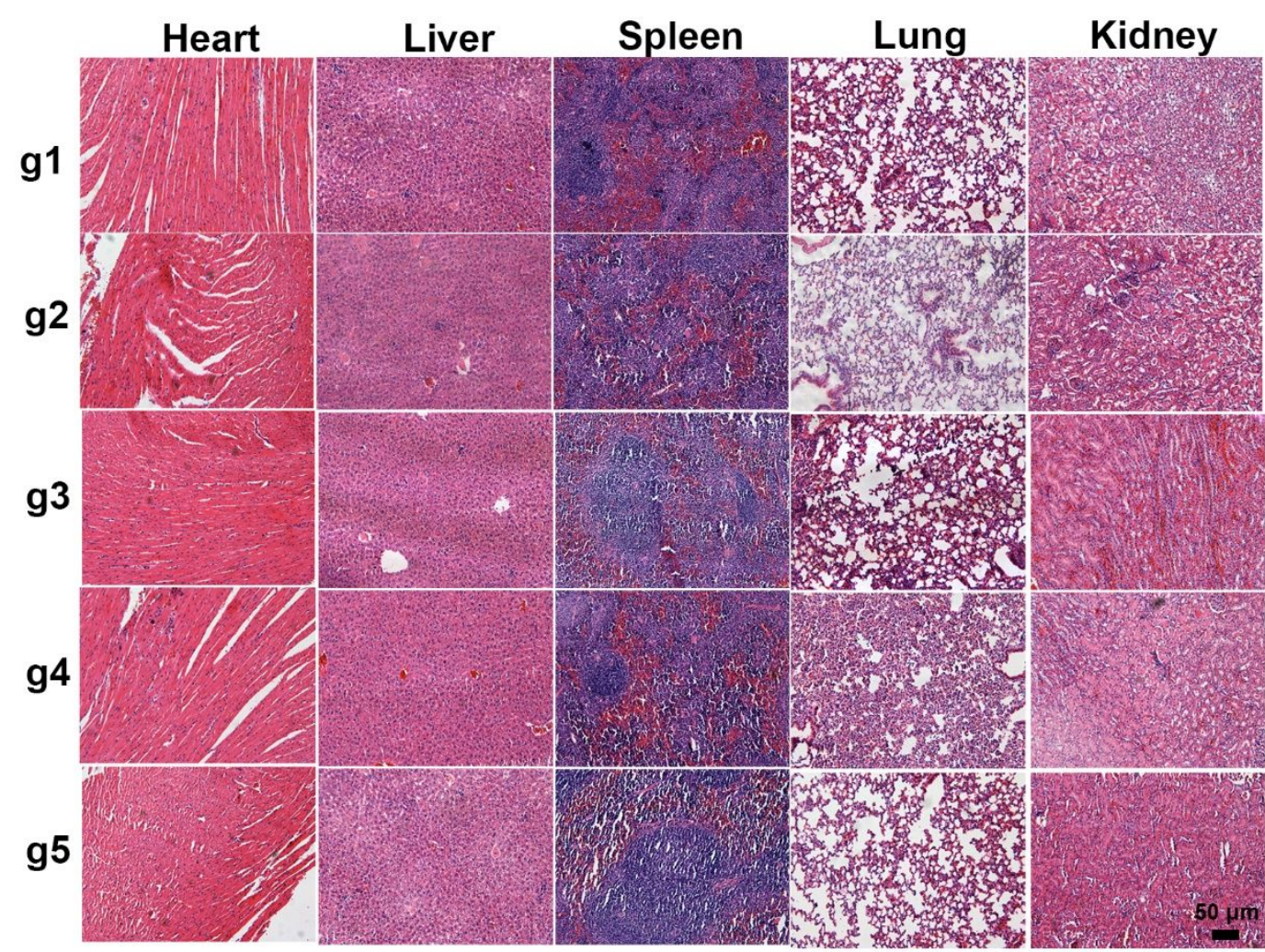

Figure S7. H\&E staining of major organs (heart, liver, spleen, lung, kidney) harvested on day 16 after injection. g1, Control; g2, PLGA@L-Arg/Gel + Laser; g3, PLGA@ICG/Gel + Laser; g4, PLGA@ICG@L-Arg + Laser; g5, PLGA@ICG@LArg/Gel + Laser; scale bar, $50 \mu \mathrm{m}$.

a
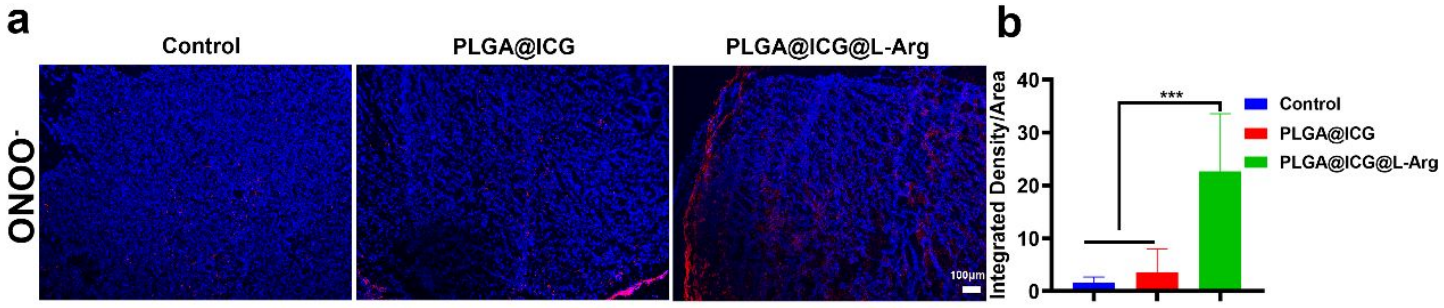

Figure S8. (a) Immunofluorescent staining of 3-nitrotyrosine (3-NT, red) in tumors in different groups. The cell nuclei (blue) were stained with DAPI; scale bar, $100 \mu \mathrm{m}$. (b) Quantitative assay of 3-N, T intensity by image J software. 
Table1. The percentage of apoptotic and necrotic cells in different groups.

\begin{tabular}{ccccc}
\hline \multirow{2}{*}{ Groups } & \multicolumn{2}{c}{-Laser } & \multicolumn{2}{c}{+ Laser } \\
\cline { 2 - 5 } & Apoptosis (\%) & Necrosis & Apoptosis (\%) & Necrosis \\
\hline Control & $9.34 \pm 1.21$ & $1.50 \pm 0.36$ & $10.56 \pm 2.35$ & $3.92 \pm 0.52$ \\
PLGA@L-Arg & $8.16 \pm 0.59$ & $1.53 \pm 0.59$ & $8.50 \pm 1.56$ & $5.83 \pm 0.36$ \\
PLGA@ICG & $9.11 \pm 2.34$ & $1.61 \pm 0.87$ & $38.29 \pm 2.05$ & $0.64 \pm 0.46$ \\
PLGA@ICG@L-Arg & $9.26 \pm 1.65$ & $1.68 \pm 0.23$ & $50.52 \pm 3.17$ & $9.10 \pm 3.69$ \\
PLGA@ICG@L- & $6.85 \pm 3.31$ & $0.61 \pm 0.34$ & $30.64 \pm 5.68$ & $12.69 \pm 2.57$ \\
\hline
\end{tabular}

